\title{
Mercury anomalies associated with three extinction events (Capitanian Crisis, Latest Permian Extinction and the Smithian/Spathian Extinction) in NW Pangea
}

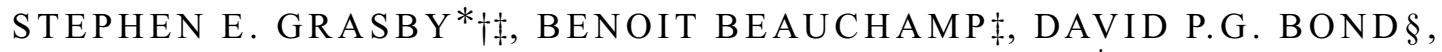 \\ PAUL B. WIGNALLף \& HAMED SANEI* \\ * Geological Survey of Canada, 3303 33rd St N.W. Calgary AB Canada, T2L 2A7 \\ $\ddagger$ Department of Geoscience, University of Calgary, Calgary AB Canada \\ $\S$ Department of Geography, Environment and Earth Sciences, University of Hull, Hull HU6 7RX, United Kingdom \\ TSchool of Earth Sciences, University of Leeds, Woodhouse Lane, Leeds LS2 9JT, United Kingdom
}

(Received 24 December 2014; accepted 21 May 2015; first published online 15 July 2015)

\begin{abstract}
Strata of Permian - Early Triassic age that include a record of three major extinction events (Capitanian Crisis, Latest Permian Extinction and the Smithian/Spathian Extinction) were examined at the Festningen section, Spitsbergen. Over the $c$. 12 Ma record examined, mercury in the sediments shows relatively constant background values of $0.005-0.010 \mu \mathrm{g} \mathrm{g}^{-1}$. However, there are notable spikes in $\mathrm{Hg}$ concentration over an order of magnitude above background associated with the three extinctions. The $\mathrm{Hg} /$ total organic carbon (TOC) ratio shows similar large spikes, indicating that they represent a true increase in $\mathrm{Hg}$ loading to the environment. We argue that these represent $\mathrm{Hg}$ loading events associated with enhanced $\mathrm{Hg}$ emissions from large igneous province (LIP) events that are synchronous with the extinctions. The $\mathrm{Hg}$ anomalies are consistent across the NW margin of Pangea, indicating that widespread mercury loading occurred. While this provides utility as a chemostratigraphic marker the $\mathrm{Hg}$ spikes may also indicate loading of toxic metals to the environment, a contributing cause to the mass extinction events.
\end{abstract}

Keywords: mercury, Early Triassic, Latest Permian Extinction, chemostratigraphy.

\section{Introduction}

Mercury $(\mathrm{Hg})$ emissions associated with the emplacement of Large Igneous Provinces (LIPs) were first recognized by Sanei, Grasby \& Beauchamp (2012), who showed a large $\mathrm{Hg}$ spike associated with the Siberian Traps eruptions. This event was coincident with the Latest Permian Extinction (LPE), the largest extinction in Earth's history that had a devastating impact on both terrestrial and marine ecosystems (Erwin, 2006). High Hg loading associated with the Siberian Traps has been supported by a similar Hg spike at the LPE boundary in Spitsbergen (Grasby et al. 2015). Recurrent Siberian Trap volcanism may also have influenced $\mathrm{Hg}$ loading during the Smithian/Spathian Extinction in the Sverdrup Basin (Grasby et al. 2013b). While these initial studies are focused on the sedimentary records from NW Pangea, the global extent of $\mathrm{Hg}$ loading related to Siberian Trap volcanism has yet to be demonstrated. However, subsequent work has shown similar $\mathrm{Hg}$ anomalies associated with other LIP events such as the Cretaceous-Palaeogene transition (Sial et al. 2013, 2014; Silva et al. 2013) related to Deccan Trap volcanism.

$\mathrm{Hg}$ is extremely toxic to life. This toxicity, combined with the ease of transport over long distances and

$\dagger$ †uthor for correspondence: sgrasby@nrcan.gc.ca persistence of $\mathrm{Hg}$ in the environment, makes modern anthropogenic mercury emissions the subject of significant global concern (AMAP, 2011). The two largest natural source of $\mathrm{Hg}$ to the environment are volcanic emissions and natural coal combustion (Pirrone et al. 2010). These sources release $\mathrm{Hg}$ to the atmosphere where it can be globally transported prior to deposition in terrestrial and marine environments. In the marine environment, organic matter and clay minerals scavenge $\mathrm{Hg}$ and transport it to the sea floor to become fixed in bottom sediments (Cranston \& Buckley, 1972; Andren \& Harriss, 1975; Lindberg, Andrenson \& Harrisson, 1975; Turner, Millward \& Le Roux, 2004; Gehrke, Blum \& Meyers, 2009). The control of primary productivity on $\mathrm{Hg}$ sequestration is shown by the close relationship between sedimentary organic matter and $\mathrm{Hg}$ in modern (Outridge et al. 2007; Stern et al. 2009; Sanei et al. 2014) as well as ancient sediments (Grasby et al. 2013b).

Modern volcanic eruptions have a significant $\mathrm{Hg}$ flux that produces global Hg anomalies (Slemr et al. 1995; Slemr \& Scheel, 1998; Schuster et al. 2002; Pyle \& Mather, 2003). Hg is sourced from both volcanic gases as well as from rock units intruded by magma (Sanei, Grasby \& Beauchamp, 2012, 2015; Grasby et al. 2015). During LIP events emission rates would greatly exceed normal Hg release (Sanei, Grasby \& Beauchamp, 2012; Grasby et al. 2015), such that the normal 


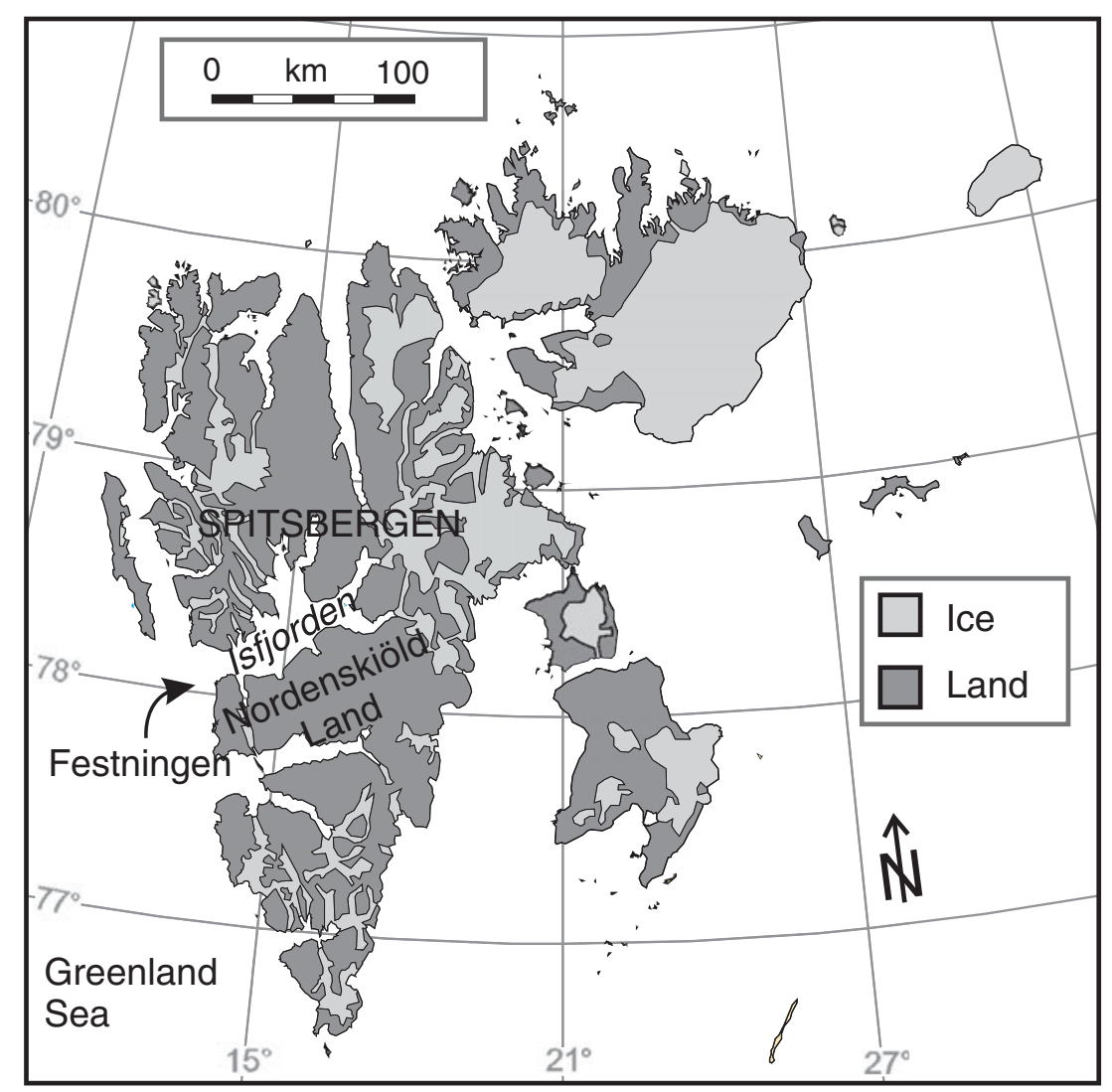

Figure 1. Map showing location of the Festningen section on Spitsbergen (base of section is located at $78.0950^{\circ} \mathrm{N}, 13.8240^{\circ} \mathrm{E}$ (WGS84 datum).

marine buffering control on $\mathrm{Hg}$ can be potentially overwhelmed (Sanei, Grasby \& Beauchamp, 2012; Grasby et al. 2013b) and be recorded as an $\mathrm{Hg}$ spike in the sediment record. For instance, Grasby et al. (2015) estimated that the Siberian Traps may have released up to $9.98 \mathrm{Gg} \mathrm{a}^{-1} \mathrm{Hg}$, or $400 \%$ above modern natural emissions. In comparision, the modern anthropogenic $\mathrm{Hg}$ emissions that roughly equal natural emissions are the subject of global concern over their impact on marine and terrestrial ecosystems. $\mathrm{Hg}$ anomalies formed at the time of LIP eruptions therefore suggest that: (1) Hg could be an effective marker in the geological record of periods of enhanced volcanic activity; and (2) LIP eruptions could potentially release toxic amounts of $\mathrm{Hg}$ to the environment. Such a scenario would add to the variety of kill mechanisms already associated with major volcanic eruptions (Keller \& Kerr, 2014), as well as providing a direct link between terrestrial and marine extinction.

To further the assessment of $\mathrm{Hg}$ in the geological record, we examined the Permian - Early Triassic record at the Festningen section in Spitsbergen (Fig. 1). This well-known location records the LPE (Grasby et al. 2015; Wignall, Morante \& Newton, 1998) as well as evidence for the earlier Capitanian Crisis (Bond et al. 2015) and Smithian/Spathian Extinction (Wignall et al. this volume), all of which have been linked to periods of major volcanic activity. Of these recent studies, the $\mathrm{Hg}$ record at Festningen has only been examined in a nar- row $(40 \mathrm{~m})$ zone straddling the LPE boundary (Grasby et al. 2015). In this paper we examine the $\mathrm{Hg}$ record at Festningen over $600 \mathrm{~m}$ of section, spanning middle Permian - Lower Triassic deposits, representing c. 12 million years of Earth's history.4.

\section{Study area}

The Festningen section is located at Kapp Starostin, Spitsbergen (Fig. 1). The $c .45^{\circ}$ east-dipping beds occur along a low-elevation sea cliff of $c .7 \mathrm{~km}$ in length. The section provides a near-continuous exposure of Carboniferous-Cenozoic strata, from Kapp Starostin to Festningsdodden, deposited in a distal broad epicontinental shelf setting on the north-western margin of Pangea (Wignall, Morante \& Newton, 1998; Stemmerik \& Worsley, 2005; Blomeier et al. 2013). During Permian time Spitsbergen was at a palaeolatitude of c. $40-45^{\circ} \mathrm{N}$ (Golonka \& Ford, 2000; Scotese, 2004).

The Kapp Starostin Formation was deposited during a period of passive subsidence that followed a major relative sea-level drop at the lower-middle Permian boundary (Blomeier et al. 2013). Deposition of widespread heterozoan carbonate (Vøringen Member) occurred during Roadian time, followed by the progradation of heterozoan carbonates and cherts over much of the Barents Shelf and Svalbard (Blomeier et al. 2013). Spitsbergen shares a similar depositional history to the palaeogeographically adjoining Sverdrup 


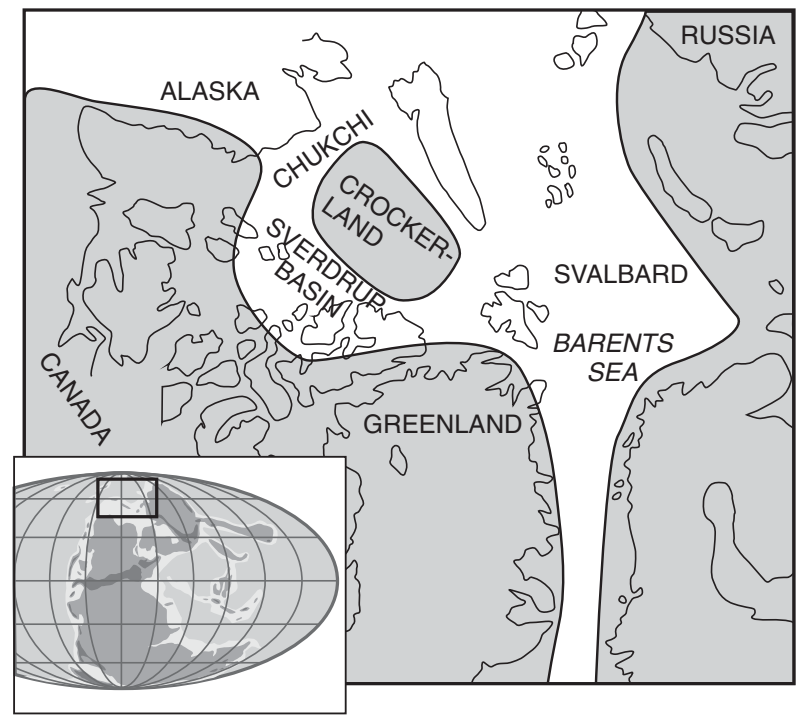

Figure 2. Palaeogeographic map (after Embry, 1992) showing relative location of Sverdrup Basin and Spitsbergen. Inset map (after Scotese, 2004) showing location on NW Pangea.

Basin (van Hauen, Degerböls and Trold Fiord formations; Beauchamp et al. 2009; Fig. 2). Approximately $40 \mathrm{~m}$ below the contact with the overlying uppermost Permian - Lower Triassic Vardebukta Formation, fossiliferous carbonates of the Kapp Starostin Formation transition to spiculitic chert of late Permian age (Blomeier et al. 2013). These later Permian chert beds are considered equivalent to the Black Stripe and Lindström formations of the Sverdrup Basin (Beauchamp et al. 2009). The Vardebukta and overlying Tvillingodden formations are dominated by shale, siltstone and minor sandstone of Early-Middle Triassic age (Mørk, Knarud \& Worsley, 1982), equivalent to the Blind Fiord Formation of the Sverdrup Basin (Embry, 1989). The basal c. 6-7 m of the Vardebukta Formation is latest Permian in age (Wignall, Morante \& Newton, 1998; Grasby et al. 2015).

\section{Methods}

A detailed sample suite was collected from $100 \mathrm{~m}$ below the Kapp Starostin/Vardebukta contact to $500 \mathrm{~m}$ above. Sample spacing varied from 1-2 $\mathrm{m}$ away from the contact to a higher density of $20-50 \mathrm{~cm}$ spacing from c. $40 \mathrm{~m}$ below to $19 \mathrm{~m}$ above the top of the Kapp Starostin Formation. Samples were recorded in metres above (positive) and below (negative) the last chert bed that defines the top of the Kapp Starostin Formation. Weathered surfaces were removed and then samples were collected from an isolated layer no greater than $2 \mathrm{~cm}$ thick. In the laboratory, any remaining weathered surfaces were removed and fresh samples were powdered by agate mortar and pestle.

Total organic carbon (TOC) was measured using Rock-Eval $6^{\odot}$, with $\pm 5 \%$ analytical error of reported value, based on repeats and reproducibility of standards run after every fifth sample (Lafargue et al. 1998). Elemental determinations were conducted on powdered samples digested in a 2:2:1:1 acid solution of $\mathrm{H}_{2} \mathrm{O}-\mathrm{HF}-\mathrm{HClO}_{4}-\mathrm{HNO}_{3}$, and subsequently analysed using a PerkinElmer Elan 9000 mass spectrometer with $\pm 2 \%$ analytical error. $\mathrm{Hg}$ was measured at GSC-Atlantic by LECO ${ }^{\circledR}$ AMA254 mercury analyser (Hall \& Pelchat, 1997) $( \pm 10 \%) . \delta^{13} \mathrm{C}_{\mathrm{org}}$ was determined on samples washed with hydrochloric acid, and rinsed with hot distilled water to remove any carbonate. $\delta^{13} \mathrm{C}$ was measured using continuousflow elemental-analysis isotope-ratio mass spectrometry, with a Finnigan Mat Delta+XL mass spectrometer interfaced with a Costech 4010 elemental analyser with combined analytical and sampling error of $\pm 0.2 \%$.

Analytical results from a total of 341 samples used in this study are provided in Table 1.

\section{Results}

\section{4.a. Organic carbon}

The organic carbon record, expressed as percent TOC, shows low values $(<1 \%$ TOC) overall (Fig. 3a). There is an increase in TOC at $-40 \mathrm{~m}$. Based on brachiopod, Sr isotopes records and magnetostratigraphy data, Bond et al. (2015) argue that the $-40 \mathrm{~m}$ level is around the middle Permian extinction level at Festningen. The TOC values decline again leading up to the LPE boundary, where there is a second brief increase in TOC just above the LPE. For the remainder of the section, TOC values in the Vardebukta Formation are $<0.2 \%$. At these low values the accuracy of Rock-Eval results decreases, however data are plotted in Figure 3a to illustrate the overall low TOC values through this interval. The TOC values increase again in the basal Tvillingodden Formation, before dropping to low values in the upper $100 \mathrm{~m}$ of section.

Rock-Eval $6^{\odot}$ results also provided information on thermal maturation and indicated that organic matter in the shales have never been heated past the upper end of the oil window, and consequently the stable isotope values of organic carbon were not thermally altered (Hayes, Kaplan \& Wedeking, 1983). The only exception is a local thermal anomaly associated with a sill emplaced at c. $19 \mathrm{~m}$ that does not extend beyond $5 \mathrm{~m}$ of the sill boundary (Grasby et al. 2015).

\section{4.b. Carbon isotope record}

Portions of the organic carbon isotope curve $\left(\delta^{13} \mathrm{C}_{\text {org }}\right)$ at the Festningen section have been reported in previous studies, including middle-upper Permian sediments (Bond et al. 2015), across the Latest Permian Extinction event (Grasby et al. 2015) and sediments of Early Triassic age (Wignall et al. this volume). The combined $\delta^{13} \mathrm{C}_{\text {org }}$ curve is provided here (Fig. 3b) for comparison with both organic carbon isotope data from NW Pangea and the global inorganic carbon 
Table 1. Geochemical data from the Festningen section. Depths are measured relative to the Latest Permian Extinction (LPE) Boundary marked by the top of the Kapp Starostin Formation. ND - not determined.

\begin{tabular}{|c|c|c|c|c|c|c|}
\hline Sample ID & $\begin{array}{c}\text { Depth relative } \\
\text { to LPE }(\mathrm{m})\end{array}$ & $\delta^{13} \mathrm{C}$ org $(\%)$ & $\mathrm{Hg}(\mathrm{ppb})$ & TOC (\%) & Mo (ppm) & $\mathrm{Al}(\%)$ \\
\hline C-556979 & -97.7 & -24.5 & 9 & 0.02 & 0.45 & 0.54 \\
\hline C- 556980 & -97.2 & -21.0 & 5 & 0.07 & 0.48 & 0.31 \\
\hline C-556981 & -95.7 & -22.2 & 4 & 0.05 & 0 & 0.59 \\
\hline C-556982 & -94.2 & ND & 5 & 0.04 & 0 & 0.58 \\
\hline C-556983 & -92.7 & -22.4 & 6 & 0.11 & 0.09 & 1.02 \\
\hline C-556984 & -91.2 & -23.0 & 2 & 0.01 & 0.05 & 0.42 \\
\hline C-556985 & -89.7 & -23.0 & 2 & 0.02 & 0.07 & 0.3 \\
\hline C-556986 & -86.7 & -25.0 & 11 & 0.22 & 0.31 & 1.59 \\
\hline C-556987 & -83.7 & -24.0 & 7 & 0.11 & 0.2 & 1.15 \\
\hline C-556988 & -80.7 & -23.7 & 5 & 0.02 & 0.08 & 0.6 \\
\hline C-556989 & -77.7 & -25.0 & 6 & 0.03 & 0.22 & 0.61 \\
\hline C-556990 & -74.7 & -23.8 & 9 & 0.12 & 0.1 & 1.55 \\
\hline C-556991 & -71.7 & -24.3 & 6 & 0.02 & 0 & 0.53 \\
\hline C-556992 & -68.7 & -24.5 & 4 & 0.02 & 0 & 0.71 \\
\hline C-556993 & -65.7 & -25.5 & 4 & 0.05 & 0 & 0.64 \\
\hline C-556994 & -62.7 & -24.4 & 4 & 0.01 & 0 & 0.85 \\
\hline C-556995 & -59.7 & -24.8 & 4 & 0.03 & 0.08 & 0.59 \\
\hline C-556996 & -56.7 & -24.6 & 4 & 0.07 & 0.09 & 0.75 \\
\hline C-556997 & -53.7 & -25.2 & 4 & 0.07 & 0.21 & 1.21 \\
\hline C-556998 & -50.7 & -25.3 & 3 & 0.10 & 0 & 1.01 \\
\hline C-556999 & -47.7 & -24.5 & 4 & 0.01 & 0 & 0.7 \\
\hline C- 557000 & -46.2 & -24.9 & 9 & 0.09 & 0.13 & 1 \\
\hline C-557001 & -44.7 & -24.5 & 6 & 0.05 & 0.13 & 1.28 \\
\hline C-557002 & -43.2 & -24.2 & 5 & 0.03 & 0.08 & 0.9 \\
\hline C-557003 & -41.7 & -23.6 & 6 & 0.08 & 0.06 & 0.45 \\
\hline C-557004 & -40.2 & -23.3 & 7 & 0.04 & 0.2 & 1.55 \\
\hline C-556572 & -27.2 & -24.3 & 28 & 0.25 & 0.53 & 2.65 \\
\hline C- 556573 & -26.7 & -24.6 & 34 & 0.44 & 0.82 & 4.18 \\
\hline C-556574 & -26.2 & -24.6 & 36 & 0.39 & 0.99 & 3.85 \\
\hline C-556575 & -25.7 & -24.4 & 22 & 0.33 & 0.57 & 3.63 \\
\hline C-556576 & -25.2 & -24.7 & 22 & 0.29 & 2.66 & 2.84 \\
\hline C-556577 & -24.7 & -24.5 & 17 & 0.22 & 1.05 & 2.6 \\
\hline C- 556578 & -24.2 & -24.4 & 35 & 0.34 & 1.83 & 3.3 \\
\hline C-556579 & -23.7 & -24.0 & 41 & 0.43 & 0 & \\
\hline C- 556580 & -23.2 & -23.8 & 34 & 0.49 & 0.34 & 3.68 \\
\hline C-556581 & -22.2 & -23.74 & 48 & 0.65 & 0.25 & 4.36 \\
\hline C-556582 & -21.7 & -23.6 & 42 & 0.69 & 0.39 & 4.43 \\
\hline C-556583 & -21.2 & -23.63 & 38 & 0.59 & 0.26 & 4.21 \\
\hline C-556584 & -20.7 & -23.87 & 30 & 0.60 & 0.48 & 4.26 \\
\hline C-556585 & -20.2 & -23.62 & 37 & 0.58 & 0.46 & 4.54 \\
\hline C-556586 & -19.7 & -23.6 & 33 & 0.55 & 0.46 & 4.67 \\
\hline C-556587 & -18.2 & -23.86 & 28 & 0.51 & 0.8 & 4.19 \\
\hline C- 556588 & -17.7 & -23.88 & 30 & 0.50 & 0.97 & 4.18 \\
\hline C-556589 & -17.2 & -23.9 & 33 & 0.57 & 1.26 & 3.97 \\
\hline C-556590 & -16.7 & -23.7 & 28 & 0.45 & 0.52 & 3.69 \\
\hline C-556591 & -16.2 & -24.0 & 29 & 0.49 & 0.7 & 4.2 \\
\hline C-556592 & -15.7 & -23.3 & 31 & 0.45 & 0.66 & 4.62 \\
\hline C-556593 & -15.2 & -24.0 & 30 & 0.53 & 0.74 & 4.08 \\
\hline C-556594 & -14.7 & -23.6 & 30 & 0.41 & 0.34 & 4.35 \\
\hline C-556595 & -14.2 & -24.1 & 32 & 0.44 & 0.33 & 4.07 \\
\hline C-556596 & -13.7 & -23.9 & 38 & 0.65 & 0.22 & 5.28 \\
\hline C-556597 & -13.2 & -23.7 & 34 & 0.50 & 0.31 & 4.7 \\
\hline C-556598 & -12.7 & -24.0 & 33 & 0.48 & 0.63 & 4.7 \\
\hline C-556599 & -12.2 & -24.3 & 37 & 0.56 & 0.57 & 5.4 \\
\hline C- 556600 & -11.7 & -24.2 & 38 & 0.64 & 0.59 & 5.58 \\
\hline C-556601 & -11.2 & -25.2 & 14 & 0.44 & 0.44 & 3.85 \\
\hline C-556602 & -10.7 & -25.1 & 13 & 0.36 & 0.69 & 4.19 \\
\hline C-556603 & -10.2 & -24.9 & 13 & 0.35 & 1.13 & 3.6 \\
\hline C-556604 & -9.7 & -24.9 & 13 & 0.21 & 0.73 & 2.5 \\
\hline C-556605 & -9.2 & -25.5 & 17 & 0.35 & 0.83 & 4.2 \\
\hline C-556606 & -8.7 & -25.4 & 14 & 0.45 & 1.01 & 3.92 \\
\hline C-556607 & -8.2 & -24.7 & 16 & 0.35 & 0.68 & 3.69 \\
\hline C-556608 & -7.7 & -25.6 & 13 & 0.32 & 0.73 & 3 \\
\hline C-556609 & -7.2 & -25.0 & 15 & 0.37 & 0.48 & 3.32 \\
\hline C-556610 & -6.7 & -24.6 & 16 & 0.37 & 0.63 & 3.2 \\
\hline C-556611 & -6.2 & -24.5 & 13 & 0.40 & 0.48 & 3.42 \\
\hline C-556612 & -5.7 & -24.3 & 13 & 0.29 & 0.29 & 3.37 \\
\hline C-556613 & -5.2 & -24.3 & 16 & 0.29 & 0.45 & 3.11 \\
\hline C-556614 & -4.7 & -24.7 & 9 & 0.45 & 0.38 & 4 \\
\hline C-556615 & -4.2 & -25.2 & 14 & 0.52 & 0.76 & 3.22 \\
\hline C-556616 & -3.7 & -25.2 & 14 & 0.24 & 0.79 & 2.89 \\
\hline C-556617 & -3.2 & -25.7 & 17 & 0.24 & 0.84 & 2.67 \\
\hline C-556618 & -3 & -25.5 & 16 & 0.22 & 0.72 & 2.77 \\
\hline
\end{tabular}


Table 1. Continued.

\begin{tabular}{|c|c|c|c|c|c|c|}
\hline Sample ID & $\begin{array}{l}\text { Depth relative } \\
\text { to LPE (m) }\end{array}$ & $\delta^{13} \mathrm{C}$ org $(\%)$ & $\mathrm{Hg}(\mathrm{ppb})$ & TOC (\%) & Mo (ppm) & $\mathrm{Al}(\%)$ \\
\hline C-556619 & -3.45 & -25.1 & 15 & 0.19 & 2.92 & 1.66 \\
\hline C-556620 & -2.8 & -25.3 & 22 & 0.40 & 0.62 & 4.48 \\
\hline C-556621 & -2.6 & -25.7 & 19 & 0.39 & 0.82 & 4.04 \\
\hline C-556622 & -2.4 & -25.9 & 18 & 0.33 & 0.96 & 3.84 \\
\hline C-556623 & -2.2 & -26.1 & 24 & 0.39 & 0.92 & 3.83 \\
\hline C-556624 & -2 & -25.6 & 19 & 0.41 & 0.6 & 3.84 \\
\hline C-556625 & -1.8 & -25.7 & 15 & 0.34 & 0.8 & 3.57 \\
\hline C-556626 & -1.6 & -25.6 & 17 & 0.46 & 0.89 & 3.9 \\
\hline C-556627 & -1.4 & -24.8 & 18 & 0.35 & 0.36 & 4.17 \\
\hline C-556628 & -1.2 & -25.6 & 24 & 0.30 & 1.14 & 3.65 \\
\hline C-556629 & -1 & -25.5 & 15 & 0.22 & 1.31 & 3.5 \\
\hline C-556630 & -0.8 & -25.1 & 18 & 0.30 & 0.75 & 3.82 \\
\hline C-556631 & -0.6 & -24.5 & 20 & 0.29 & 0.41 & 3.43 \\
\hline C-556632 & -0.4 & -24.2 & 21 & 0.39 & 0.77 & 4.33 \\
\hline C-556633 & -0.2 & -25.3 & 16 & 0.23 & 0.57 & 3.42 \\
\hline C-556634 & 0 & -25.5 & 20 & 0.33 & 1.37 & 3.33 \\
\hline C-556635 & 1 & -29.0 & 29 & 0.28 & 0.48 & 7.98 \\
\hline C-556636 & 1.2 & -29.6 & 43 & 0.24 & 1.07 & 8.21 \\
\hline C-556637 & 1.4 & -29.1 & 48 & 0.19 & 1.34 & 8.69 \\
\hline C-556638 & 1.6 & -28.8 & 33 & 0.13 & 1.21 & 8.45 \\
\hline C-556639 & 1.8 & -28.6 & 27 & 0.14 & 0.28 & 8.25 \\
\hline C-556640 & 2 & -28.7 & 26 & 0.11 & 0.52 & 8.52 \\
\hline C-556641 & 2.2 & -28.8 & 16 & 0.10 & 0.14 & 8.57 \\
\hline C-556642 & 2.4 & -28.9 & 20 & 0.09 & 0.16 & 8.57 \\
\hline C-556643 & 2.6 & -30.1 & 30 & 0.13 & 0.46 & 9.98 \\
\hline C-556644 & 2.8 & -30.4 & 17 & 0.10 & 0.14 & 7.85 \\
\hline C-556645 & 2.9 & -30.3 & 25 & 0.08 & 0.46 & 9.5 \\
\hline C-556646 & 3.1 & -30.8 & 25 & 0.12 & 0.17 & 7.87 \\
\hline C-556647 & 3.3 & -31.0 & 27 & 0.22 & 0.16 & 8.2 \\
\hline C-556648 & 3.5 & -32.8 & 41 & 0.40 & 0.55 & 7.96 \\
\hline C-556649 & 3.7 & -32.6 & 43 & 0.45 & 0.96 & 8.09 \\
\hline C-556650 & 3.9 & -32.5 & 42 & 0.43 & 0.57 & 8.21 \\
\hline C-556651 & 4.1 & -33.5 & 77 & 0.31 & 4.4 & 9.75 \\
\hline C-556652 & 4.3 & -32.8 & 60 & 0.48 & 1.59 & 7.87 \\
\hline C-556653 & 4.4 & -32.6 & 74 & 0.27 & 0.63 & 8.05 \\
\hline C-556654 & 5.4 & -32.5 & 62 & 0.50 & 1.43 & 7.99 \\
\hline C-556655 & 5.6 & -32.2 & 53 & 0.33 & 1.23 & 8.19 \\
\hline C-556656 & 5.8 & -33.3 & 86 & 0.42 & 2.39 & 9.91 \\
\hline C-556657 & 6 & -32.6 & 69 & 0.44 & 0.76 & 9.66 \\
\hline C-556658 & 6.2 & -33.2 & 69 & 0.31 & 0.57 & 9.77 \\
\hline C-556659 & 6.4 & -33.6 & 60 & 0.39 & 1.04 & 8.47 \\
\hline C-556660 & 6.6 & -33.3 & 53 & 0.28 & 0.48 & 10.3 \\
\hline C-556661 & 6.8 & -33.2 & 46 & 0.26 & 0.35 & 10.97 \\
\hline C-556662 & 7 & -32.8 & 53 & 0.34 & 0.44 & 11.28 \\
\hline C-556663 & 7.2 & -32.6 & 51 & 0.34 & 0.52 & 10.92 \\
\hline C-556664 & 7.4 & -33.0 & 50 & 0.31 & 0.39 & 10.76 \\
\hline C-556665 & 7.6 & -32.9 & 81 & 0.44 & 2.76 & 10.02 \\
\hline C-556666 & 7.8 & -32.9 & 77 & 0.56 & 5.03 & 8.99 \\
\hline C-556667 & 8 & -32.3 & 82 & 0.71 & 3.91 & 9.56 \\
\hline C-556668 & 8.15 & -32.7 & 89 & 0.51 & 5.2 & 7.92 \\
\hline C-556669 & 8.2 & -33.7 & 134 & 1.03 & 20.65 & 8.74 \\
\hline C-556670 & 8.4 & -33.0 & 88 & 0.63 & 10.16 & 7.12 \\
\hline C-556671 & 8.6 & -33.5 & 100 & 0.61 & 26.37 & 7.02 \\
\hline C-556672 & 8.8 & -33.6 & 57 & 0.38 & 4.51 & 9.62 \\
\hline C-556673 & 9 & -33.8 & 57 & 0.41 & 3.67 & 9.2 \\
\hline C-556674 & 9.2 & -32.1 & 25 & 0.08 & 1.58 & 6.99 \\
\hline C-556675 & 9.4 & -33.3 & 50 & 0.46 & 2.77 & 9.55 \\
\hline C-556676 & 9.6 & -32.8 & 38 & 0.25 & 0.59 & 10.06 \\
\hline C-556677 & 9.8 & -33.1 & 26 & 0.30 & 0.96 & 9.54 \\
\hline C-556678 & 10 & -32.9 & 47 & 0.23 & 0.62 & 10.87 \\
\hline C-556679 & 10.2 & -33.2 & 35 & 0.30 & 1.02 & 10.63 \\
\hline C-556680 & 10.4 & -33.0 & 30 & 0.29 & 1.92 & 10.99 \\
\hline C-556681 & 10.6 & -32.8 & 40 & 0.49 & 1.98 & 9.53 \\
\hline C-556682 & 10.8 & -31.6 & 19 & 0.14 & 1.42 & 6.76 \\
\hline C-556683 & 11 & -31.8 & 23 & 0.17 & 1.4 & 9.02 \\
\hline C-556684 & 11.2 & -27.4 & 74 & 1.02 & 1.41 & 9.1 \\
\hline C-556685 & 11.4 & -31.8 & 34 & 0.17 & 2.14 & 8.21 \\
\hline C-556686 & 11.6 & -32.1 & 23 & 0.21 & 0.83 & 9.45 \\
\hline C-556687 & 11.8 & -32.2 & 31 & 0.25 & 1.51 & 9.31 \\
\hline C-556688 & 12 & -32.6 & 48 & 0.39 & 3.08 & 9.09 \\
\hline C-556689 & 12.2 & -32.6 & 56 & 0.55 & 2.9 & 9.23 \\
\hline C-556690 & 12.4 & -31.3 & 19 & 0.09 & 1.28 & 6.7 \\
\hline C-556691 & 12.6 & -33.1 & 34 & 0.29 & 2.55 & 9.55 \\
\hline C-556692 & 12.8 & -32.7 & 35 & 0.15 & 3.04 & 6.67 \\
\hline
\end{tabular}


Table 1. Continued.

\begin{tabular}{|c|c|c|c|c|c|c|}
\hline Sample ID & $\begin{array}{l}\text { Depth relative } \\
\text { to LPE (m) }\end{array}$ & $\delta^{13} \mathrm{C}$ org $(\% 0)$ & $\mathrm{Hg}(\mathrm{ppb})$ & TOC (\%) & Mo (ppm) & $\mathrm{Al}(\%)$ \\
\hline C-556693 & 13 & -33.1 & 45 & 0.33 & 7.18 & 7.38 \\
\hline C-556694 & 13.2 & -32.8 & 47 & 0.29 & 4 & 7.1 \\
\hline C-556695 & 13.05 & -33.9 & 41 & 0.48 & 12.34 & 9.75 \\
\hline C-556696 & 13.4 & -32.7 & 34 & 0.30 & 4.13 & 7.19 \\
\hline C-556697 & 13.6 & -33.6 & 42 & 0.50 & 4.96 & 7.1 \\
\hline C-556698 & 13.8 & -33.3 & 43 & 0.48 & 3.89 & 7.31 \\
\hline C-556699 & 14 & -32.4 & 35 & 0.17 & 2.55 & 7.44 \\
\hline C- 556700 & 14.2 & -33.4 & 27 & 0.31 & 3.45 & 4.54 \\
\hline C-556701 & 14.4 & -33.5 & 24 & 0.26 & 3.5 & 4.57 \\
\hline C-556702 & 14.8 & -32.8 & 33 & 0.45 & 2.31 & 7.7 \\
\hline C- 556703 & 15.3 & -32.4 & 15 & 0.18 & 1.71 & 7.25 \\
\hline C-556704 & 15.8 & -31.2 & 10 & 0.10 & 0.37 & 7.99 \\
\hline C-556705 & 16.3 & -31.8 & 7 & 0.28 & 1.53 & 7.82 \\
\hline C-556706 & 16.8 & -32.4 & 1 & 0.35 & 1.27 & 7.98 \\
\hline C-556707 & 17.3 & -32.4 & 1 & 0.19 & 3.08 & 8.2 \\
\hline C-556708 & 17.8 & -25.1 & 0.43 & 0.00 & 3.34 & 7.79 \\
\hline C-556709 & 18.8 & ND & 0.49 & 0.01 & 3.01 & 7.72 \\
\hline C-556710 & 20.3 & -31.9 & 0.36 & 0.19 & 3.51 & 7.98 \\
\hline C-556711 & 21.8 & -32.6 & 4 & 0.16 & 1.32 & 8.08 \\
\hline C-556712 & 23.3 & -32.5 & 18 & 0.64 & 3.42 & 7.49 \\
\hline C-556713 & 24.8 & -33.0 & 20 & 0.42 & 2.66 & 7.43 \\
\hline C-556714 & 26.3 & -32.7 & 22 & 0.50 & 2.96 & 7.07 \\
\hline C-556715 & 27.8 & -32.7 & 26 & 0.54 & 2.9 & 7.03 \\
\hline C-556716 & 29.3 & -32.8 & 25 & 0.40 & 1.21 & 7.42 \\
\hline C-556717 & 30.8 & -32.3 & 24 & 0.32 & 1.36 & 7.1 \\
\hline C-556718 & 32.3 & -32.8 & 31 & 0.73 & 3.33 & 7.09 \\
\hline C-556719 & 33.8 & -32.1 & 34 & 0.57 & 2.31 & 7.16 \\
\hline C-556720 & 35.3 & -31.3 & 23 & 0.35 & 1.04 & 7.42 \\
\hline C-556721 & 36.8 & -31.8 & 18 & 0.49 & 3.17 & 7.3 \\
\hline C-556722 & 38.3 & -31.7 & 9 & 0.23 & 0.4 & 6.65 \\
\hline C-556723 & 39.8 & -31.3 & 9 & 0.26 & 0.8 & 7.43 \\
\hline C-556724 & 41.3 & -31.3 & 16 & 0.30 & 0.42 & 7.29 \\
\hline C-556725 & 44.3 & -31.5 & 22 & 0.49 & 0.75 & 7.38 \\
\hline C-556726 & 47.3 & -31.4 & 29 & 0.62 & 0.71 & 6.84 \\
\hline C-556727 & 50.3 & -31.1 & 27 & 0.45 & 0.36 & 7.16 \\
\hline C-556728 & 53.3 & -29.2 & 9 & 0.05 & 0.55 & 4.44 \\
\hline C-556729 & 56.3 & -29.9 & 16 & 0.12 & 0.42 & 6.64 \\
\hline C-556730 & 59.3 & -29.7 & 11 & 0.15 & 0.33 & 6.94 \\
\hline C-556731 & 62.3 & -28.7 & 10 & 0.15 & 0.17 & 7.56 \\
\hline C-556732 & 65.3 & -29.2 & 8 & 0.12 & 0.29 & 6.92 \\
\hline C-556733 & 68.3 & -29.8 & 5 & 0.07 & 0.28 & 6.51 \\
\hline C-556734 & 71.3 & -29.6 & 8 & 0.09 & 0.59 & 6.67 \\
\hline C-556735 & 74.3 & -29.6 & 8 & 0.10 & 0.46 & 6.01 \\
\hline C-556736 & 77.3 & -29.5 & 5 & 0.06 & 0.31 & 6.88 \\
\hline C-556737 & 80.3 & -29.4 & 7 & 0.07 & 0.29 & 6.66 \\
\hline C-556738 & 83.3 & -29.2 & 7 & 0.06 & 0.26 & 6.43 \\
\hline C-556739 & 86.3 & -28.6 & 4 & 0.04 & 0.27 & 6.58 \\
\hline C- 556740 & 89.3 & -29.1 & 14 & 0.11 & 0.64 & 6.84 \\
\hline C-556741 & 92.3 & -28.5 & 5 & 0.05 & 0.19 & 6.32 \\
\hline C-556742 & 95.3 & -27.9 & 8 & 0.13 & 0.23 & 6.24 \\
\hline C-556743 & 98.3 & -28.5 & 5 & 0.05 & 0.28 & 6.36 \\
\hline C- 556744 & 101.3 & -28.0 & 4 & 0.11 & 0.24 & 5.53 \\
\hline C-556745 & 104.3 & -28.3 & 8 & 0.05 & 0.25 & 5.57 \\
\hline C-556746 & 107.3 & -27.6 & 3 & 0.03 & 0.16 & 6.66 \\
\hline C-556747 & 110.3 & -28.3 & 5 & 0.12 & 0.25 & 6.04 \\
\hline C-556748 & 113.3 & -27.6 & 4 & 0.02 & 0.18 & 5.84 \\
\hline C-556749 & 116.3 & -27.8 & 12 & 0.03 & 0.45 & 4.94 \\
\hline C-556750 & 117.8 & -28.7 & 16 & 0.15 & 0.85 & 6.42 \\
\hline C-556751 & 119.3 & -28.3 & 22 & 0.16 & 0.45 & 6.54 \\
\hline C-556752 & 122.3 & -28.9 & 16 & 0.07 & 0.51 & 4.1 \\
\hline C-556753 & 125.3 & -27.6 & 14 & 0.06 & 0.3 & 5.12 \\
\hline C-556754 & 128.3 & -26.7 & 9 & 0.04 & 0.3 & 5.95 \\
\hline C- 556755 & 134.3 & -27.4 & 10 & 0.06 & 0.22 & 6.44 \\
\hline C-556756 & 137.3 & -27.7 & 4 & 0.02 & 0.16 & 6.36 \\
\hline C-556757 & 140.3 & -26.8 & 8 & 0.04 & 0.27 & 6.19 \\
\hline C-556758 & 143.3 & -28.3 & 30 & 0.28 & 0.82 & 6.06 \\
\hline C- 556759 & 146.3 & -27.2 & 21 & 0.09 & 0.27 & 5.97 \\
\hline C-556760 & 149.3 & -26.7 & 10 & 0.08 & 0.29 & 6.92 \\
\hline C-556761 & 152.3 & -26.7 & 16 & 0.10 & 0.19 & 6.55 \\
\hline C-556762 & 153.3 & -26.0 & 7 & 0.03 & 0.25 & 6.03 \\
\hline C- 556763 & 154.8 & -26.0 & 22 & 0.11 & 0.38 & 6.86 \\
\hline C-556764 & 157.8 & -26.9 & 10 & 0.07 & 0.18 & 6.32 \\
\hline C-556765 & 160.8 & -27.4 & 17 & 0.12 & 0.27 & 7.81 \\
\hline C-556766 & 163.8 & -26.8 & 7 & 0.02 & 0.41 & 6.26 \\
\hline
\end{tabular}


Table 1. Continued.

\begin{tabular}{|c|c|c|c|c|c|c|}
\hline Sample ID & $\begin{array}{l}\text { Depth relative } \\
\text { to LPE (m) }\end{array}$ & $\delta^{13} \mathrm{C}$ org $(\%)$ & $\mathrm{Hg}(\mathrm{ppb})$ & TOC (\%) & Mo (ppm) & $\mathrm{Al}(\%)$ \\
\hline C-556767 & 166.8 & -26.3 & 9 & 0.11 & 0.29 & 6.92 \\
\hline C-556768 & 169.8 & -26.1 & 9 & 0.05 & 0.22 & 6.75 \\
\hline C-556769 & 182.3 & -26.5 & 4 & 0.04 & 0.19 & 5.03 \\
\hline C-556770 & 196.8 & -24.7 & 7 & 0.06 & 0.18 & 5.23 \\
\hline C-556771 & 199.8 & -25.7 & 7 & 0.03 & 0.14 & 6.66 \\
\hline C-556772 & 202.8 & -25.7 & 4 & 0.02 & 0.13 & 6.27 \\
\hline C-556773 & 205.8 & -26.0 & 4 & 0.01 & 0.18 & 4.37 \\
\hline C-556774 & 208.8 & -26.1 & 5 & 0.07 & 0.16 & 5.78 \\
\hline C-556775 & 214.8 & -26.0 & 3 & 0.01 & 0.08 & 5.68 \\
\hline C-556776 & 217.8 & -26.6 & 5 & 0.02 & 0.14 & 6.88 \\
\hline C-556777 & 220.8 & -27.1 & 7 & 0.04 & 0.11 & 6.86 \\
\hline C-556778 & 223.8 & -27.4 & 5 & 0.02 & 0.15 & 6.06 \\
\hline C-556779 & 226.8 & -26.8 & 5 & 0.09 & 0.16 & 5.99 \\
\hline C-556780 & 229.8 & -27.1 & 7 & 0.02 & 0.18 & 5.1 \\
\hline C-556781 & 232.8 & -28.7 & 10 & 0.05 & 0.14 & 5.21 \\
\hline C-556782 & 235.8 & -27.4 & 10 & 0.03 & 0.41 & 4.33 \\
\hline C-556783 & 238.8 & -27.4 & 6 & 0.03 & 0.18 & 6.22 \\
\hline C-556784 & 241.8 & -27.7 & 8 & 0.06 & 0.24 & 6.65 \\
\hline C-556785 & 244.8 & -28.1 & 11 & 0.06 & 0.29 & 5.71 \\
\hline C-556786 & 247.8 & -29.2 & 8 & 0.06 & 0.2 & 5.61 \\
\hline C-556787 & 250.8 & -29.4 & 7 & 0.06 & 0.2 & 6.31 \\
\hline C-556788 & 253.8 & -28.5 & 5 & 0.05 & 0.19 & 5.81 \\
\hline C-556789 & 256.8 & -31.1 & 11 & 0.15 & 0.32 & 6.17 \\
\hline C-556790 & 260.8 & -29.7 & 12 & 0.09 & 0.31 & 6.36 \\
\hline C-556791 & 263.8 & -29.4 & 9 & 0.06 & 0.44 & 5.57 \\
\hline C-556792 & 266.8 & -31.4 & 14 & 0.13 & 0.3 & 6.08 \\
\hline C-556793 & 269.8 & -31.6 & 13 & 0.19 & 0.43 & 5.85 \\
\hline C-556794 & 272.8 & -31.5 & 15 & 0.16 & 0.39 & 6.14 \\
\hline C-556795 & 275.8 & -28.7 & 5 & 0.05 & 0.14 & 6.15 \\
\hline C-556796 & 278.8 & -30.2 & 6 & 0.10 & 0.15 & 5.37 \\
\hline C-556797 & 281.8 & -30.7 & 5 & 0.05 & 0.18 & 5.13 \\
\hline C-556798 & 284.8 & -31.4 & 12 & 0.11 & 0.62 & 5.93 \\
\hline C-556799 & 290.8 & -30.1 & 5 & 0.06 & 0.31 & 5.87 \\
\hline C-556800 & 293.8 & -31.2 & 15 & 0.15 & 1.08 & 6.6 \\
\hline C-556801 & 296.8 & -31.3 & 10 & 0.15 & 1.09 & 6.19 \\
\hline C-556802 & 299.8 & -32.5 & 54 & 0.50 & 7.52 & 6.65 \\
\hline C-556803 & 302.8 & -32.7 & 55 & 0.30 & 10.08 & 5.56 \\
\hline C-556804 & 309.8 & -32.4 & 43 & 0.32 & 5.94 & 5.81 \\
\hline C-556805 & 312.8 & -32.9 & 36 & 0.23 & 8.15 & 4.67 \\
\hline C-556806 & 315.8 & -32.8 & 65 & 0.48 & 11.74 & 6 \\
\hline C-556807 & 318.8 & -30.6 & 38 & 0.43 & 1.46 & 6.31 \\
\hline C-556808 & 321.8 & -30.7 & 32 & 0.46 & 1.77 & 5.93 \\
\hline C-556809 & 324.8 & -30.6 & 28 & 0.57 & 1.54 & 5.72 \\
\hline C-556810 & 327.8 & -29.9 & 16 & 0.28 & 0.57 & 5.41 \\
\hline C-556811 & 330.8 & -29.6 & 23 & 0.49 & 0.73 & 6.23 \\
\hline C-556812 & 333.8 & -30.3 & 30 & 0.46 & 1.55 & 6.23 \\
\hline C-556813 & 336.8 & -29.6 & 32 & 0.61 & 1.88 & 6.43 \\
\hline C-556814 & 339.8 & -29.3 & 11 & 0.20 & 0.48 & 5.07 \\
\hline C-556815 & 342.8 & -28.6 & 26 & 0.62 & 7.48 & 5.08 \\
\hline C-556816 & 345.8 & -28.7 & 26 & 0.91 & 8.42 & 6.32 \\
\hline C-556817 & 348.8 & -28.6 & 18 & 0.62 & 3.14 & 6.27 \\
\hline C-556818 & 351.8 & -28.6 & 8 & 0.22 & 1.44 & 4.98 \\
\hline C-556819 & 354.8 & -29.1 & 19 & 0.93 & 1.33 & 6.25 \\
\hline C-556820 & 357.8 & -29.4 & 19 & 0.95 & 0.99 & 5.79 \\
\hline C-556821 & 360.8 & -29.3 & 21 & 1.13 & 0.83 & 6.51 \\
\hline C-556822 & 363.8 & -29.1 & 14 & 0.66 & 0.52 & 6.06 \\
\hline C-556823 & 366.8 & -29.6 & 13 & 0.43 & 2.08 & 4.21 \\
\hline C-556824 & 369.8 & -30.2 & 26 & 0.86 & 4.3 & 6.68 \\
\hline C-556825 & 372.8 & -30.5 & 24 & 0.80 & 3.07 & 6.7 \\
\hline C-556826 & 375.8 & -30.6 & 19 & 0.43 & 2.47 & 5.69 \\
\hline C-556827 & 378.8 & -30.7 & 17 & 0.38 & 2.11 & 5.7 \\
\hline C-556828 & 381.8 & -30.5 & 15 & 0.25 & 2.61 & 5.96 \\
\hline C-556829 & 387.8 & -30.7 & 12 & 0.30 & 2.45 & 5.74 \\
\hline C-556830 & 390.8 & -30.6 & 13 & 0.28 & 0.8 & 5.75 \\
\hline C-556831 & 393.8 & -30.4 & 8 & 0.23 & 0.76 & 4.31 \\
\hline C-556832 & 396.8 & -30.6 & 10 & 0.28 & 0.44 & 4.86 \\
\hline C-556833 & 399.8 & -30.8 & 13 & 0.42 & 0.79 & 5.86 \\
\hline C-556834 & 402.8 & -30.5 & 11 & 0.34 & 0.54 & 5.84 \\
\hline C-556835 & 405.8 & -30.1 & 6 & 0.15 & 0.36 & 3.72 \\
\hline C-556836 & 408.8 & -30.8 & 8 & 0.25 & 1.1 & 4.54 \\
\hline C-556837 & 411.8 & -30.8 & 12 & 0.28 & 1.41 & 4.96 \\
\hline C-556838 & 414.8 & -31.5 & 20 & 0.53 & 3.72 & 6.7 \\
\hline C-556839 & 417.8 & -31.3 & 13 & 0.33 & 1.99 & 6 \\
\hline C-556840 & 420.8 & -30.9 & 13 & 0.21 & 1.96 & 5.37 \\
\hline
\end{tabular}


Table 1. Continued.

\begin{tabular}{lcccccc}
\hline Sample ID & $\begin{array}{c}\text { Depth relative } \\
\text { to LPE }(\mathrm{m})\end{array}$ & $\delta^{13} \mathrm{C}$ org $(\%)$ & $\mathrm{Hg}(\mathrm{ppb})$ & $\mathrm{TOC}(\%)$ & Mo (ppm) & Al (\%) \\
\hline C-556841 & 423.8 & -31.5 & 12 & 0.37 & 1.17 & 5.94 \\
C-556842 & 426.8 & -30.4 & 6 & 0.11 & 1.2 & 3.86 \\
C-556843 & 429.8 & -31.4 & 11 & 0.34 & 3.49 & 5.8 \\
C-556844 & 432.8 & -31.0 & 9 & 0.26 & 2.28 & 3.79 \\
C-556845 & 435.8 & -31.7 & 17 & 0.54 & 6.92 & 5.49 \\
C-556846 & 438.8 & -31.8 & 11 & 0.25 & 6.32 & 4.44 \\
C-556847 & 441.8 & -31.2 & 6 & 0.19 & 1.7 & 3.85 \\
C-556848 & 444.8 & -30.3 & 4 & 0.11 & 1.99 & 3.37 \\
C-556849 & 447.8 & -31.2 & 6 & 0.20 & 5.56 & 4.08 \\
C-556850 & 450.8 & -30.9 & 6 & 0.22 & 6.11 & 3.81 \\
C-556851 & 453.8 & -30.5 & 8 & 0.13 & 2.78 & 2.99 \\
C-556852 & 456.8 & -31.1 & 10 & 0.16 & 3.47 & 3.68 \\
C-556853 & 459.8 & -29.7 & 4 & 0.07 & 0.66 & 4.23 \\
C-556854 & 462.8 & -30.4 & 6 & 0.10 & 1.8 & 3.73 \\
C-556855 & 468.3 & -28.4 & 7 & 0.11 & 0.25 & 4.19 \\
C-556856 & 475.3 & -29.6 & 5 & 0.10 & 1.23 & 4.87 \\
C-556857 & 479.8 & -30.2 & 7 & 0.11 & 0.45 & 4.62 \\
C-556858 & 482.8 & -30.3 & 7 & 0.14 & 0.55 & 4.04 \\
C-556859 & 485.8 & -29.5 & 8 & 0.14 & 0.75 & 4.93 \\
C-556860 & 488.8 & -28.5 & 3 & 0.05 & 0.22 & 3.78 \\
C-556861 & 491.8 & -29.8 & 7 & 0.12 & 0.42 & 4.09 \\
C-556862 & 499.8 & -30.0 & 7 & 0.24 & 0.63 & 3.98 \\
\hline
\end{tabular}
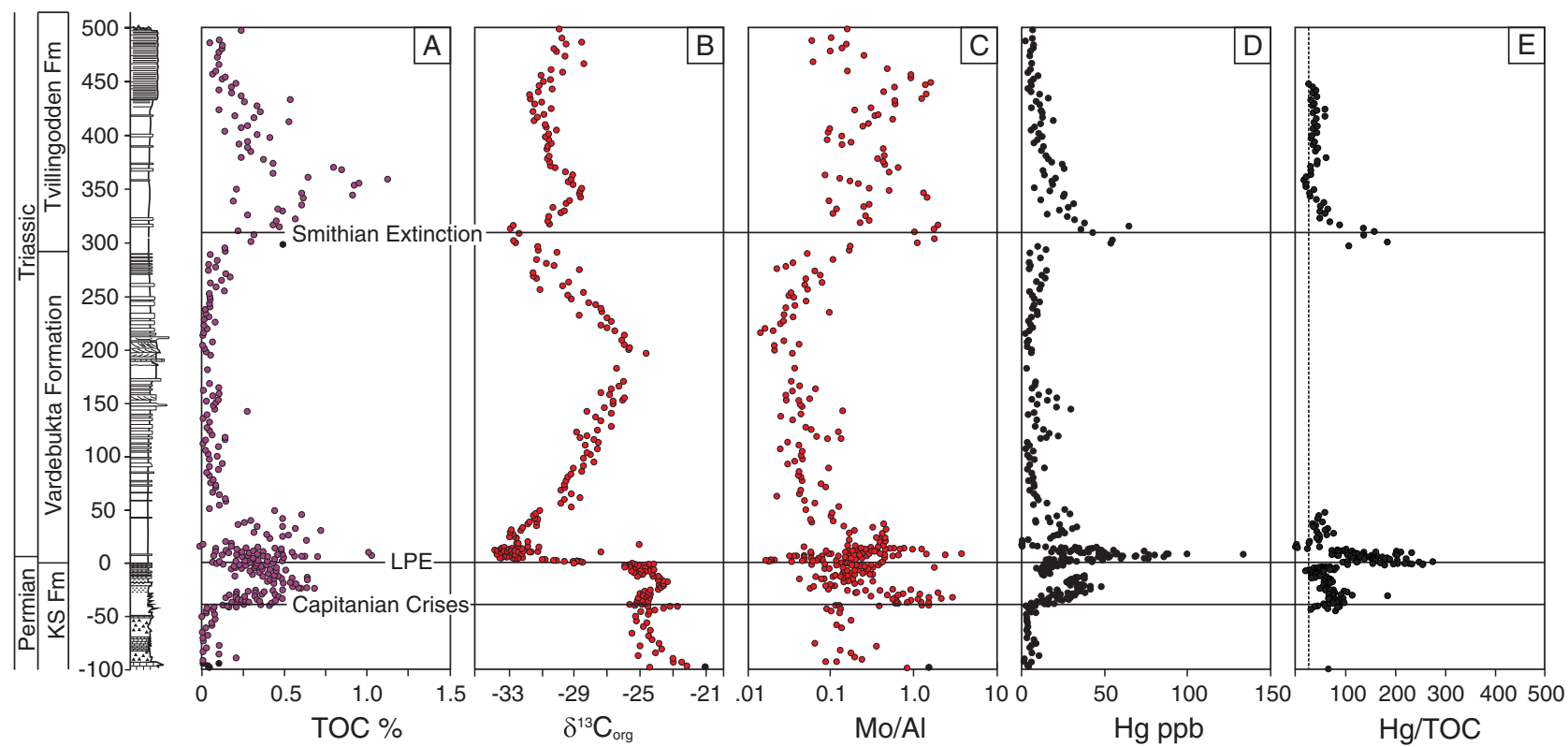

Figure 3. (Colour online) Plots of geochemical data from Festningen, showing: (a) percent total organic carbon (TOC); (b) carbon isotope values for organic carbon; (c) Mo normalized by Al; (d) $\mathrm{Hg}$ values; and (e) Hg normalized by TOC (the $\mathrm{Hg}$ / TOC values are only shown for values of TOC $>0.2 \%$; below that value Rock-Eval analyses provide less accurate results that are magnified in calculated ratios).

isotope record. The $\delta^{13} \mathrm{C}_{\text {org }}$ data from Festningen show initially relatively stable values of $c$. $-24 \%$ through the middle-upper Permian sediments, with a relatively minor negative shift at $c$. $-40 \mathrm{~m}$. At the top of the Kapp Starostin Formation, carbon isotope values show the onset of a pronounced $10 \%$ negative shift through the basal Vardebukta Formation sediments (Grasby et al. 2015). After this point there is a progressive recovery through the next $200 \mathrm{~m}$, followed by another progressive negative shift to a minimum at $c .+300 \mathrm{~m}$ which is followed by an unstable period through the remainder of the measured section (Wignall et al. this volume).

\section{4.c. Molybdenum}

Similar to the carbon isotope record, trace element data have been shown as a proxy for anoxia for the three separate portions of the section previously studied (Bond et al. 2015; Grasby et al. 2015; Wignall et al. this volume). For reference, the composite curve of Mo normalized to $\mathrm{Al}$ is shown through the entire interval (Fig. 3c). Results show relatively low values at the base of the section with a significant spike in $\mathrm{Mo} / \mathrm{Al}$ at $c$. $40 \mathrm{~m}$, which coincides with the shift to higher TOC values and minor negative shift in $\delta^{13} \mathrm{C}_{\text {org }}$. Above the LPE boundary there is a second spike in Mo/Al that 
initiates c. $5 \mathrm{~m}$ after the main LPE event. This is followed by a gradual decline in $\mathrm{Mo} / \mathrm{Al}$ until c. $200 \mathrm{~m}$, coincident with the reversal in the trend of carbon isotopes. The $\mathrm{Mo} / \mathrm{Al}$ values progressively increase again through the next $100 \mathrm{~m}$ until $+300 \mathrm{~m}$, where peak $\mathrm{Mo} / \mathrm{Al}$ ratios are coincident with the minimum in $\delta^{13} \mathrm{C}_{\text {org }}$ values. $\mathrm{Mo} / \mathrm{Al}$ values then become highly variable for the rest of the measured section.

\section{4.d. Mercury}

$\mathrm{Hg}$ values for the studied section, reported here for the first time, are plotted in Figure $3 \mathrm{~d}$. $\mathrm{Hg}$ values are $0.005-0.010 \mu \mathrm{g} \mathrm{g}^{-1}$ in the basal $60 \mathrm{~m}$ of section and then show a shift above $-40 \mathrm{~m}$ to values up to $0.050 \mu \mathrm{g} \mathrm{g}^{-1}$, followed by a subsequent decline to values of $c .0 .020 \mu \mathrm{g} \mathrm{g}^{-1}$ in the $10 \mathrm{~m}$ just below the LPE boundary. At the LPE there is a significant spike in $\mathrm{Hg}$ to the highest values observed in the section $\left(>0.130 \mu \mathrm{g} \mathrm{g}^{-1}\right)$. Hg concentrations drop rapidly to background values of $0.005-0.010 \mu \mathrm{g} \mathrm{g}^{-1}$ through the remainder of the section. The only exception is a brief spike at $c$. $+300 \mathrm{~m}$, where $\mathrm{Hg}$ concentrations up to $0.065 \mu \mathrm{g} \mathrm{g}^{-1}$ are observed.

In both marine and freshwater environments, dissolved $\mathrm{Hg}$ has been shown to have a strong affinity for organic matter (OM) (Mason, Reinfelder \& Morel, 1996; Gagnon, Pelletier \& Mucci, 1997; Benoit et al. 2001; Han et al. 2006; Gehrke, Blum \& Meyers, 2009). Grasby et al. (2013b) have also shown that OM strongly controls $\mathrm{Hg}$ sequestration over geological time. Along with absolute concentrations, the $\mathrm{Hg} / \mathrm{TOC}$ ratio is plotted in Figure 3e. However, TOC values are too low to be considered accurate for parts of the section, where Rock-Eval analyses cannot accurately resolve concentrations $<0.2 \% \mathrm{TOC}$; as a result, only samples with TOC $>0.2 \%$ are plotted as lower accuracy can greatly affect the calculated ratio. These data show a general trend where the $\mathrm{Hg} / \mathrm{TOC}$ ratio has constant low values through the section. The exceptions to this are the large spikes in $\mathrm{Hg} / \mathrm{TOC}$ values that occur at the LPE boundary as well as smaller shifts that occur at $-40 \mathrm{~m}$ as well as at $c .+300 \mathrm{~m}$. These increases in $\mathrm{Hg} / \mathrm{TOC}$ ratio are consistent with zones where there are large spikes in absolute $\mathrm{Hg}$ concentration. $\mathrm{Hg}$ values are low outside these three levels.

\section{Discussion}

\section{5.a. Carbon isotope chemostratigraphy}

The $\delta^{13} \mathrm{C}$ record through sediments of Permian - Early Triassic age shows significant shifts that are comparable to those observed in the Sverdrup Basin (Grasby et al. 2013a) as well as with inorganic $\delta^{13} \mathrm{C}$ trends from records in the Panthalassa (Horacek, Koike \& Richoz, 2009) and the Tethys (Payne et al. 2004; Horacek, Brandner \& Abart, 2007). This demonstrates that Festningen records global variation in biogeochemical cycles through this time interval. These can be used as a chemostratigraphic tool to support both regional and global correlation.

Bond et al. (2015) argued that the minor negative carbon anomaly at $-40 \mathrm{~m}$ correlates with the Capitanian Crisis. The LPE event, as marked by the loss of chert forming siliceous sponges, is characterized by the onset of a large negative shift in $\delta^{13} \mathrm{C}_{\text {org }}$ that reaches a minimum in the basal Vardebukta Formation (Wignall, Morante \& Newton, 1998; Grasby et al. 2015). Above the Kapp Starostin Formation, Wignall et al. (this volume) show that the next negative low point at c. $300 \mathrm{~m}$ is correlative with the end-Smithian Substage. This is also consistent with comparison to the $\delta^{13} \mathrm{C}_{\text {org }}$ record from the Smithian stratotype (Grasby et al. 2013a), as illustrated in Figure 4. This makes the low in carbon isotope values equivalent to the Smithian/Spathian Extinction event that was associated with renewed Siberian Trap volcanism and rapid global temperature increase (Brayard et al. 2006; Orchard, 2007; Xie et al. 2010; Sun et al. 2012).

\section{5.b. Mercury deposition}

The Hg record at Festningen shows a relatively constant background value through the majority of the succession analysed $\left(<0.020 \mu \mathrm{g} \mathrm{g}^{-1}\right)$. However, notable spikes in $\mathrm{Hg}$ concentration occur coincident with the three extinction levels represented in this section (Capitanian Crisis, LPE and Smithian/Spathian Extinction). Of these three spikes the most significant occurs at the LPE boundary. Aside from the three most prominent $\mathrm{Hg}$ spikes, there is also a slight increase in the upper Dienerian portion of the section. It is interesting to note that these prominent $\mathrm{Hg}$ spikes are all associated with shifts to lower $\delta^{13} \mathrm{C}$ values. In addition, there is a general association of higher $\mathrm{Hg}$ concentrations associated with high Mo/Al values (Fig. 2). While this may suggest increased $\mathrm{Hg}$ sequestration associated with changes to more anoxic environments, a plot of $\mathrm{Mo} / \mathrm{Al}$ versus $\mathrm{Hg}$ reveals that there is no correlation (Fig. 5). Despite the general relationship, the data suggest that anoxia has no direct influence on $\mathrm{Hg}$ sequestration in sediments.

Given the low organic matter content throughout much of the Lower Triassic portion of the studied section, reliable $\mathrm{Hg} / \mathrm{TOC}$ ratios are not always possible to obtain. For values $<0.2 \%$ TOC, inaccuracies in measurement can lead to magnified errors and highly variable $\mathrm{Hg} / \mathrm{TOC}$ values that are not reflective of natural conditions; these are therefore not shown here. However, for TOC values $>0.2 \%$, the $\mathrm{Hg} / \mathrm{TOC}$ ratios for the Festningen section have relatively constant background values (vertical dashed line in Fig. 3e). These results are consistent with $\mathrm{Hg} / \mathrm{TOC}$ through the Lower Triassic Smithian stratotype, Sverdrup Basin (Grasby et al. $2013 b$ ), indicating a general background level of $\mathrm{Hg}$ sequestration into sediment that is largely controlled by organic matter deposition.

The notable exceptions to this background Hg deposition are spikes in both total $\mathrm{Hg}$ concentrations as well as $\mathrm{Hg} / \mathrm{TOC}$ levels at the three extinction levels (Fig. 3). 


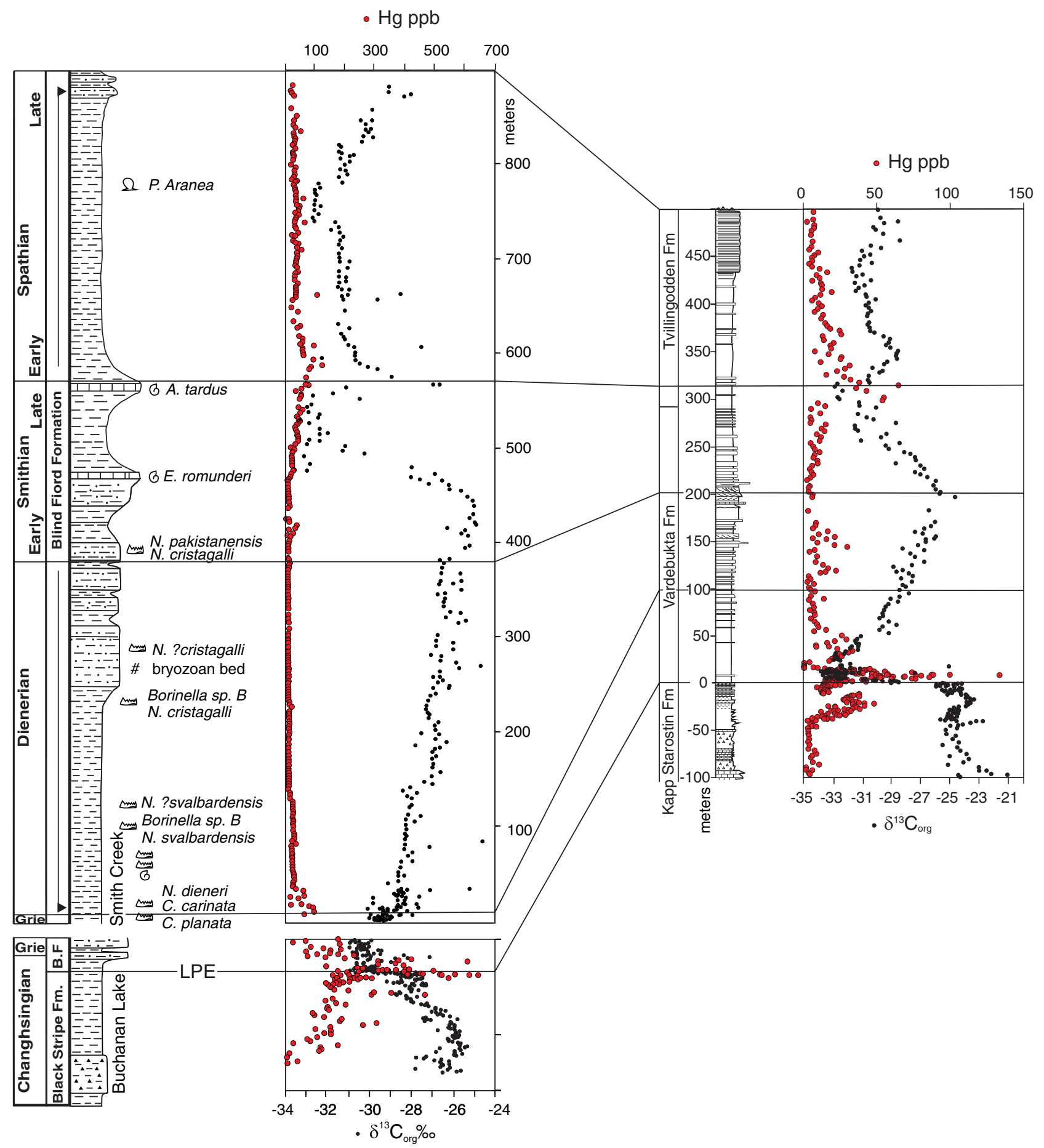

Figure 4. (Colour online) Correlation of carbon isotope and Hg records from the Smithian stratotype (left side; Grasby et al. 2013a) with record from Festningen (right side; this study).

Previously Sanei, Grasby \& Beauchamp (2012) and Grasby et al. (2013b) argued that $\mathrm{Hg}$ anomalies in the geological record are related to massive $\mathrm{Hg}$ emissions associated with periods of major volcanic eruptions. Our results from Festningen provide support for this hypothesis, showing that over the $c .12$ Ma record there are three prominent $\mathrm{Hg}$ anomalies superimposed on background $\mathrm{Hg}$ concentrations. In each case these $\mathrm{Hg}$ spikes are associated with global extinction events that have previously been tied to LIP eruptions: Capitanian
Crisis (Emeishan eruptions); LPE (Siberian Traps); and Smithian/Spathian Extinction (renewed Siberian Traps) (Paton et al. 2010; Xie et al. 2010; Bond \& Wignall, 2014).

On a regional perspective the $\mathrm{Hg}$ spikes observed at Festningen closely correspond to those in the Sverdrup Basin (Fig. 4). This indicates that these periods of anomalous $\mathrm{Hg}$ deposition are regional in scope, and suggests periods of enhanced $\mathrm{Hg}$ deposition over broad areas. 


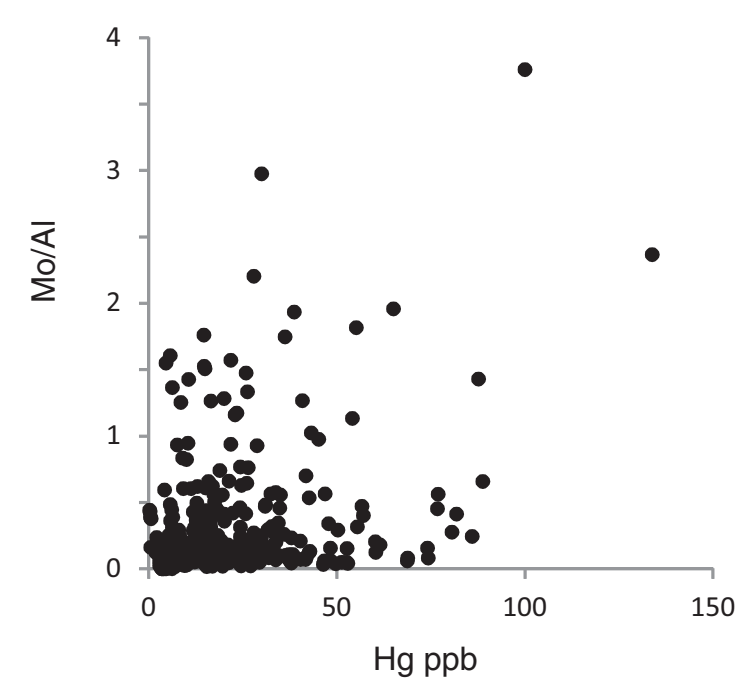

Figure 5. Plot of the $\mathrm{Mo} / \mathrm{Al}$ ratio versus $\mathrm{Hg}$ concentration.

\section{Conclusions}

The organic carbon isotope records from the Festningen section show trends through sediments of late Permian - Early Triassic age that closely correspond to those of the Sverdrup Basin, as well as the global inorganic carbon record. These results illustrate that NW Pangea records perturbations to the global carbon cycle. It was previously demonstrated that $\mathrm{Hg}$ is an excellent proxy for periods of major volcanic activity in the geological record (Sanei, Grasby \& Beauchamp, 2012; Grasby et al. 2013b; Sial et al. 2013). The Festningen section records a general constant background of $\mathrm{Hg}$ deposition through time. However, there are notable spikes in $\mathrm{Hg}$ concentration as well as in the $\mathrm{Hg} / \mathrm{TOC}$ ratio that correspond to periods of mass extinction (Capitanian Crisis, the LPE event and the Smithian/Spathian Extinction), all of which have been associated with LIP events. Our results therefore support the use of $\mathrm{Hg}$ as a marker for LIP eruptions. What remains uncertain is what role such $\mathrm{Hg}$ release could have on ecosystems. $\mathrm{Hg}$ is certainly one of the most toxic elements for life, and enhanced $\mathrm{Hg}$ flux from large igneous events would likely have a global impact on both the terrestrial and marine realm. Our results show that there are consistent records of $\mathrm{Hg}$ spikes associated with LIP events and mass extinctions across NW Pangea. The global nature of these records remains to be demonstrated. If they are more widely distributed, then $\mathrm{Hg}$ release could have played a role as a significant extinction mechanism throughout Earth's history.

Acknowledgements. This research was made possible by Karsten Piepjohn of Bundesanstalt für Geowissenschaftern und Rohstoffe, Geozentrum. M. Parson assisted with Hg analyses and R. Stewart with Rock-Eval analyses. DB acknowledges financial support from NERC Advanced Fellowship grant NE/J01799X/1 and the Research Executive Agency for Marie Curie IEF grant FP7-PEOPLE-2011-IEF-300455. PW acknowledges support from NERC grant NE/I015817/1.

\section{References}

AMAP. 2011. AMAP Assessment 2011: Mercury in the Arctic. Oslo, Norway: Arctic Monitoring and Assessment Programme(AMAP).

ANDREN, A. W. \& HARRISS, R. C. 1975. Observations on the association between mercury and organic matter dissolved in natural waters. Geochimica et Cosmochimica Acta 39(9), 1253-58.

Beauchamp, B., Henderson, C. M. B., Grasby, S. E., Gates, L., BeAtTy, T., UtTing, J. \& JAmes, N. P. 2009. Late Permian sedimentation in the Sverdrup Basin, Canadian Arctic: the Lindström and Black Stripe formations. Canadian Society of Petroleum Geology Bulletin 57, 167-91.

Benoit, J. M., Mason, R. P., Gilmour, C. C. \& Aiken, G. R. 2001. Constants for mercury binding by dissolved organic matter isolates from the Florida Everglades. Geochimica et Cosmochimica Acta 65(24), 4445-51.

BlomeIER, D., DustirA, A. M., Forke, H. \& SCHEIBNER, C. 2013. Facies analysis and depositional environments of a storm-dominated, temperate to cold, mixed siliceouscarbonate ramp: the Permian Kapp Starostin Formation in NE Svalbard. Norwegian Journal of Geology 93, 7598.

Bond, D. P. G. \& Wignall, P. B. 2014. Large igneous provinces and mass extinctions: An update. In Volcanism, Impacts, and Mass Extinctions: Causes and Effects (eds G. Keller \& A. C. Kerr). Geological Society of America, Special Papers 505, doi:10.1130/2014.2505(02).

Bond, D. P. G., Wignall, P. B., JOAChimski, M., Sun, Y., SAVOV, I., GRASBY, S. E., BEAUCHAMP, B. \& BlOMEIER, D. P. G. 2015. An abrupt extinction in the Middle Permian (Capitanian) of the Boreal Realm (Spitsbergen). Geological Society of America Bulletin, published online 14 April 2015, doi: 10.1130/B31216.1.

Brayard, A., Bucher, H., Escarguel, G., Fluteau, F., Bourquin, S. \& GALFETTI, T. 2006. The Early Triassic ammonoid recovery: Paleoclimatic significance of diversity gradients. Palaeogeography, Palaeoclimatology, Palaeoecology 239(3-4), 374-95.

Cranston, R. E. \& Buckley, D. E. 1972. Mercury pathways in a river and estuary. Environmental Science \& Technology 6(3), 274-78.

EMBRY, A. 1989. Correlation of Upper Palaeozoic and Mesozoic sequences between Svalbard, Canadian Arctic Archipelago, and northern Alaska. In Correlation in $\mathrm{Hy}$ drocarbon Exploration (ed. J. D. Collinson), pp. 89-98. Netherlands: Springer.

EMBRY, A. F. 1992. Crockerland: The Northwest source area for the Sverdrup Basin, Canadian Arctic Islands. In Arctic Geology and Petroleum Potential (eds T. O. Vorren, E. Bergsager, Ø. A. Dahl-Stamnes, E. Holter, B. Johansen, E. Lie \& T. B. Lund), pp. 205-16. Amsterdam: Elsevier.

ERwIN, D. H. 2006. Extinction. How Life on Earth Nearly Ended 250 million years ago. New Jersey: Princeton University Press.

Gagnon, C., Pelletier, É. \& Mucci, A. 1997. Behaviour of anthropogenic mercury in coastal marine sediments. Marine Chemistry 59(1-2), 159-76.

Gehrke, G. E., Blum, J. D. \& Meyers, P. A. 2009. The geochemical behavior and isotopic composition of $\mathrm{Hg}$ in a mid-Pleistocene western Mediterranean sapropel. Geochimica et Cosmochimica Acta 73(6), 1651-65.

GolonKA, J. \& FORD, D. 2000. Pangean (Late Carboniferous-Middle Jurassic) paleoenvironment and 
lithofacies. Palaeogeography, Palaeoclimatology, Palaeoecology 161, 1-34.

Grasby, S. E., Beauchamp, B., Bond, D. P. G., Wignall, P. B., Talavera, C., Galloway, J. M., Piepjohn, K., REINHARDT, L. \& BLOMEIER, D. 2015. Progressive environmental deterioration in NW Pangea leading to the Latest Permian Extinction. Geological Society of America Bulletin, published online 14 April 2015, doi: 10.1130/B31197.1.

Grasby, S. E., Beauchamp, B., Embry, A. F. \& Sanei, H. 2013a. Recurrent Early Triassic ocean anoxia. Geology 41, 175-78.

Grasby, S. E., SANei, H., Beauchamp, B. \& Chen, Z. $2013 b$. Mercury deposition through the Permo-Triassic Biotic Crisis. Chemical Geology 351, 209-16.

Hall, G. \& Pelchat, P. 1997. Evaluation of a direct solid sampling atomic absorption spectrometer for the trace determination of mercury in geological samples. Analyst 122(9), 921-24.

Han, S., Gill, G. A., Lehman, R. D. \& Choe, K.-Y. 2006. Complexation of mercury by dissolved organic matter in surface waters of Galveston Bay, Texas. Marine Chemistry 98(2-4), 156-66.

Hayes, J. M., Kaplan, I. R. \& Wedeking, K. W. 1983. Precambrian organic geochemistry, preservation of the record. In Earth's Earliest Biosphere: Its Origin and Evolution (ed. J. W. Schopf), pp. 92-132. Princeton NJ: Princeton University Press.

HoraceK, M., BrandNER, R. \& ABART, R. 2007. Carbon isotope record of the $\mathrm{P} / \mathrm{T}$ boundary and the Lower Triassic in the Southern Alps: Evidence for rapid changes in storage of organic carbon. Palaeogeography, Palaeoclimatology, Palaeoecology 252(1-2), 347-54.

HoraceK, M., KoIKE, T. \& RichOZ, S. 2009. Lower Triassic $\delta^{13} \mathrm{C}$ isotope curve from shallow-marine carbonates in Japan, Panthalassa realm: Confirmation of the Tethys $\delta^{13} \mathrm{C}$ curve. Journal of Asian Earth Sciences 36(6), 48190.

Keller, G. \& KerR, A. C. (eds) 2014. Volcanism, Impacts, and Mass Extinctions: Causes and Effects. Boulder, CO: Geological Society of America.

Lafargue, E., Espitalité, J., Marquis, F. \& Pillot, D. 1998. Rock-Eval 6 applications in hydrocarbon exploration, production and soil contamination studies. Revue de L'institut Francais du Petrole 53(4), 421-37.

LindBerg, S. E., ANDrenson, A. W. \& Harrisson, R. C. 1975. Geochemistry of mercury in the estuarine environment. In Estuarine Research. Chemistry, Biology and the Estuarine System (ed. E. L. Cronin). New York: Academic Press.

Mason, R. P., Reinfelder, J. R. \& Morel, F. M. M. 1996. Uptake, toxicity, and trophic transfer of mercury in a coastal diatom. Environmental Science \& Technology 30(6), 1835-45.

Mørk, A., KNARUd, R. \& Worsley, D. 1982. Depositional and diagenetic environments of the Triassic and Lower Jurassic succession of Svalbard. In Arctic Geology and Geophysics: Proceedings of the Third International Symposium on Arctic Geology (eds A. F. Embry \& H. R. Balkwill), pp. 371-98. Calgary: Canadian Society of Petroleum Geologists.

ORCHARD, M. J. 2007. Conodont diversity and evolution through the latest Permian and Early Triassic upheavals. Palaeogeography, Palaeoclimatology, Palaeoecology 252(1-2), 93-117.

Outridge, P. M., Sanei, H., Stern, G. A., Hamilton, P. B. \& GOODARZI, F. 2007. Evidence for control of mercury accumulation in sediments by variations of aquatic primary productivity in Canadian High Arctic lakes. Environmental Science \& Technology 41, 5259-65.

PAton, M. T., IVANOV, A. V., Fiorentini, M. L., MCNaughton, N. J., MudrovsKa, I., ReZnitskit, L. Z. \& Demonterova, E. I. 2010. Late Permian and Early Triassic magmatic pulses in the Angara-Taseeva syncline, Southern Siberian Traps and their possible influence on the environment. Russian Geology and Geophysics 51(9), 1012-20.

Payne, J. L., Lehrmann, D. J., Wei, J., Orchard, M. J., SCHRAG, D. P. \& KNOLL, A. H. 2004. Large perturbations of the carbon cycle during recovery from the EndPermian extinction. Science 305, 506-9.

Pirrone, N., Cinnirella, S., Feng, X., Finkelman, R. B., Friedli, H. R., Leaner, J., Mason, R., MuKherJee, A. B., Stracher, G. B., Streets, D. G. \& Telmer, K. 2010. Global mercury emissions to the atmosphere from anthropogenic and natural sources. Atmospheric Chemistry and Physics Discussions 10, 4719-52.

Pyle, D. M. \& MATHER, T. A. 2003. The importance of volcanic emissions for the global atmospheric mercury cycle. Atmospheric Environment 37(36), 5115-24.

SaneI, H., Grasby, S. E. \& Beauchamp, B. 2012. Latest Permian mercury anomalies. Geology 40(1), 63-6.

SANEI, H., Grasby, S. E. \& Beauchamp, B. 2015. Contaminants in marine sedimentary deposits from coal fly ash during the Latest Permian Extinction (Chapter 5). In: Environmental Contaminants: Using Natural Archives to Track Sources and Long-Term Trends of Pollution (J. M. Blais, M. R. Rosen \& J. P. Smol, eds), pp. 89100. Springer, Developments in Paleoenvironmental Research vol. 18 .

SAnei, H., Outridge, P. M., Stern, G. A. \& Macdonald, R. W. 2014.Classification of mercury-labile organic matter relationships in lake sediments. Chemical Geology 373, 87-92.

Schuster, P. F., Krabbenhoft, D. P., NAftz, D. L., Cecil, L. D., Olson, M. L., Dewild, J. F., Susong, D. D., GreEN, J. R. \& ABBotT, M. L. 2002. Atmospheric mercury deposition during the last 270 years: a glacial ice core record of natural and anthropogenic sources. Environmental Science \& Technology 36(11), 2303-10.

SCOTESE, C. R. 2004. A continental drift flipbook. Journal of Geology 112, 729-41.

Sial, A. N., Chen, J., Lacerda, L. D., Peralta, S., Gaucher, C., Frei, R., Cirilli, S., Ferreira, V. P., Marquillas, R. A., Barbosa, J. A., Pereira, N. S. \& Belmino, I. K. C. 2014. High-resolution Hg chemostratigraphy: a contribution to the distinction of chemical fingerprints of the Deccan volcanism and Cretaceous-Paleogene Boundary impact event. Palaeogeography, Palaeoclimatology, Palaeoecology 414, 98 115.

Sial, A. N., Lacerda, L. D., Ferreira, V. P., Frei, R., Marquillas, R. A., Barbosa, J. A., GaUcher, C., Windmöller, C. C. \& PereirA, N. S. 2013. Mercury as a proxy for volcanic activity during extreme environmental turnover: The Cretaceous-Paleogene transition. Palaeogeography, Palaeoclimatology, Palaeoeco$\log y$ 387, 153-64.

Silva, M. V. N., Sial, A. N., Barbosa, J. A., Ferreira, V. P., Neumann, V. H. \& De Lacerda, L. D. 2013. Carbon isotopes, rare-earth elements and mercury geochemistry across the $\mathrm{K}-\mathrm{T}$ transition of the Paraíba Basin, northeastern Brazil. In Isotopic Studies in Cretaceous Research (A.-V. Bojar, M. C. Melinte-Dobrinescu \& J. Smit, eds), pp. 85-104. Geological Society, London, Special Publications no. 382. 
SLEMR, F., JUNKERMANN, W., SCHMIDT, R. W. H. \& SLADKOVIC, R. 1995. Indication of change in global and regional trends of atmospheric mercury concentrations. Geophysical Research Letters 22(16), 2143-6.

Slemr, F. \& ScheEl, H. E. 1998. Trends in atmospheric mercury concentrations at the summit of the Wank mountain, Southern Germany. Atmospheric Environment 32(5), 845-53.

STEMMERIK, L. \& WORSLEY, D. 2005. 30 years on: Arctic Upper Palaeozoic stratigraphy, depositional evolution and hydrocarbon prospectivity. Norsk Geologisk Tidsskrift 85, 151-68.

Stern, G. A., Sanei, H., Roach, P., Delaronde, J. \& OUTRIDGE, P. M. 2009. Historical interrelated variations of mercury and aquatic organic matter in lake sediment cores from a subarctic lake in Yukon, Canada: further evidence toward the algal-mercury scavenging hypothesis. Environmental Science \& Technology 43, 7684-90.

Sun, Y., JoAChimski, M. M., Wignall, P. B., Yan, C., Chen, Y., JIANG, H., WANG, L. \& LAI, X. 2012. Lethally hot temperatures during the Early Triassic greenhouse. Science 338(6105), 366-70.

Turner, A., Millward, G. E. \& Le RouX, S. M. 2004. Significance of oxides and particulate organic matter in controlling trace metal partitioning in a contaminated estuary. Marine Chemistry 88(3-4), 179-92.

Wignall, P. B., Bond, D. P. G., Sun, Y., Grasby, S. E., Beauchamp, B., JoAchimski, M. \& Blomeier, D. This volume. Ultra-shallow marine anoxia in an Early Triassic storm-dominated clastic ramp (Spitsbergen) and the suppression of benthic radiation. Geological Magazine.

Wignall, P. B., Morante, R. \& Newton, R. 1998. The Permo-Triassic transition in Spitsbergen: $\delta^{13} \mathrm{C}_{\text {org }}$ chemostratigraphy, Fe and $\mathrm{S}$ geochemistry, facies, fauna and trace fossils. Geological Magazine 135, 4762.

Xie, S., Pancost, R. D., Wang, Y., Yang, H., Wignall, P. B., LuO, G., JiA, C. \& CHEN, L. 2010. Cyanobacterial blooms tied to volcanism during the 5 m.y. PermoTriassic biotic crisis. Geology 38(5), 447-50. 